\title{
Commissioning and validation of the electron Monte Carlo dose calculation at extended source to surface distance from a medical linear accelerator
}

\author{
T. Arunkumar', C. Varatharaj', M. Ravikumar ${ }^{3}$, K. M. Ganesh ${ }^{3}$, S. Sathiyan ${ }^{4}$, Shwetha B ${ }^{2}$ \\ ${ }^{1}$ Research Scholar, ${ }^{2}$ Assistant Professor of Radiation Physics, ${ }^{3}$ Professor of Radiation Physics, ${ }^{4}$ Associate Professor of \\ Radiation Physics; all are attached with Department of Radiation Physics, Kidwai Memorial Institute of Oncology, Hosur \\ Road, Bangalore, India.
}

Address for correspondence: Dr C. Varatharaj, Email: drvaratharaj@gmail.com

\begin{abstract}
Introduction: Radiotherapy is one of the major modality for cancer management playing curative, adjuvant, and palliative and sometimes has an alternative role to chemotherapy. Radiotherapy is practiced in two ways viz. External beam therapy and Brachytherapy. Electron beam therapy is widely used in the management of cancers. An electron beam is characterized by a finite range of penetration with a rapid dose fall off towards a slowly decaying $\mathrm{x}$-ray background as the electrons traverse through tissues. The electron monte carlo (eMC) dose calculation algorithm for eclipse treatment system has been introduced by Varian Medical systems. The algorithm is commissioned and validated by comparing percentage depth dose (PDD) and gamma index. Methods: Percentage depth dose curves were generated for all the energies for $4 \times 4 \mathrm{~cm}^{2}$ and $10 \times 10 \mathrm{~cm}^{2}$ field sizes. The depth of maximum dose $\left(\mathrm{R}_{100}\right)$, therapeutic depth $\left(\mathrm{R}_{85}\right)$, depth of $50 \%$ isodose $\left(\mathrm{R}_{50}\right)$ and the relative surface $\left(\mathrm{D}_{\mathrm{s}}\right)$ were compared with the measured and calculated PDD curves. Results: The eMC calculated fluence and measured fluence were compared for all the energies and cones at nominal source to surface distance and extended distances. For $4 \times 4 \mathrm{~cm}^{2}$ field size the maximum shift in $\mathrm{R}_{100}$ was $5 \mathrm{~mm}, \mathrm{R}_{85}$ was $1.9 \mathrm{~mm}, \mathrm{R}_{50}$ was $0.9 \mathrm{~mm}$ and the variation in the relative surface $\left(\mathrm{D}_{\mathrm{S}}\right)$ was about $25 \mathrm{Gamma}$ analysis shows excellent agreements with greater than $98 \%$ of the pixels passing the gamma requirements. Conclusion: We have successfully commissioned and validated the electron monte carlo dose calculation at extended source to surface distance.
\end{abstract}

Key words: Eclipse, Electron Monte Carlo, Fluence, Gamma value.

\section{Introduction}

Radiotherapy, is one of the principal modalities used in the treatment of cancer, the other two being surgery and chemotherapy. For over 50 years, electron beam therapy has been an important radiation therapy modality in the management of cancers. This broad acceptance is attributed in part to the unique characteristics and the easy accessibility of electron beams to practitioners. Electron beams are produced from the linear accelerators that can be found in most radiation oncology centers. In certain clinical situations, electron beam treatments are performed at extended source to surface distance (SSD $100-120 \mathrm{~cm}$ ) due to the limitation of the electron collimator/cone and the site of

Manuscript received $14^{\text {th }}$ June 2016

Reviewed: $24^{\text {th }}$ June 2016

Author Corrected: $4^{\text {th }}$ July 2016

Accepted for Publication $16^{\text {th }}$ July 2016 treatment. Additionally sometimes a larger field size or increased penumbra may be needed for a particular radiation treatment. Sites such as head and neck, vulva and groin and breast may require extended distances for electron beam treatments as body anatomy may obstruct the positioning of the electron applicator. Dosimetry and beam characteristics at extended SSDs and small field sizes are dependent on the collimator/cone design, mode of electron production and beam energy.

Electron beam treatments are carried out not only at nominal source to surface distance $(\mathrm{SSD}=100 \mathrm{Cm})$ but also at the extended SSDs. Most of the planning systems use empirical methods and pencil beam algorithms which has limitations in predicting the percentage depth dose and optimum fluence at extended SSDs. The electron monte carlo (eMC) dose calculation 
algorithm is available in the Varian eclipse treatment planning system. The algorithm consists of

1) Electron transport/dose deposition model (transport model, macro monte carlo method [1]) performing the transport and dose deposition caused by the electron in the patient.

2) Electron beam phase-space model (Initial Phase model, IPS) describing the electrons that emerge from the treatment head of the linear accelerator.

The eMC has six user selectable parameters for individual calculations: calculation grid size, accuracy, maximum number of particle histories, random number generator seed, smoothing method and smoothing level [2]. To attain accurate calculations and consistency within a reasonable amount of time, the calculation used in the study are done with $2.5 \mathrm{~mm}$ calculation grid size, 3D Gaussian smoothing method and medium level smoothing.

The accuracy of implementation of this algorithm was investigated by several groups [1-4]. The beam data required for commissioning of this algorithm has been measured and the dosimetric quantities were validated by comparing the PDDs, absolute dose and gamma index for nominal and extended SSDs.

\section{Materials and Methods}

\section{a) Configuration of the eMC Algorithm:}

The following beam data measurements for the full open field and energy/applicator combination were carried out to configure the eMC algorithm [5]

1)Percentage depth dose curve in air at source to phantom distance $(\mathrm{SPD}=100 \mathrm{~cm})$ for each energy, without an applicator, collimator jaws wide open $\left(40 \times 40 \mathrm{~cm}^{2}\right)$.

2)Beam profile in air at $95 \mathrm{~cm}$ source to detector distance (SDD) for all the energies (normalized to 1).

3)Percentage depth dose curve in water at source to phantom distance $(\mathrm{SPD}=100 \mathrm{~cm})$ for each energy, with an applicator.

4) Absolute dose in water expressed in (cGy/MU) at the calibration point in the depth dose curve (measured at reference depth).

These measured data's were converted in to w2cad file and then imported into the eclipse beam configuration task. The eMC algorithm generates a calculated depth dose curve taking this input data.

\section{b) Validation}

A water equivalent phantom was created in eclipse treatment planning system. The following measurements were made to evaluate the Varian eclipse electron monte Carlo (eMC) algorithm performance. Depth dose curves were generated for all the energies $(6,9,12,16$ and $20 \mathrm{MeV})$ at $100,102,106$ and $110 \mathrm{~cm}$ SSD's for $4 \times 4 \mathrm{~cm}^{2}$ and $10 \times 10 \mathrm{~cm}^{2}$ field sizes. Similarly isodose distributions were generated for all the energies at 100,102, 106 and $110 \mathrm{~cm}$ SSD's for $4 \times 4 \mathrm{~cm}^{2}, 6 \times 6$ $\mathrm{cm}^{2}, 10 \times 10 \mathrm{~cm}^{2}, 15 \times 15 \mathrm{~cm}^{2}$ and $20 \times 20 \mathrm{~cm}^{2}$ field sizes and for a irregular cut-out $\left(6.5 \times 9 \mathrm{~cm}^{2}\right.$ used for patient treatment). The measurements were done with the Clinac 2100 - DHX linear accelerator (Varian Medical Systems Palo Alto, CA). The PDD curves were measured using a scanning water tank system (RFA 300, Scanditronix Medical AB with Omni Pro 6 software). Initial eMC plans were created in eclipse for each cone size and energy combination without normalization point. Dose maximum values were determined in these plans by using Eclipse vertical dose profile tool along the central axis. The plans were normalized to $100 \%$ at their respective $\mathrm{d}_{\max }$ for analysis. The depth of maximum dose $\left(\mathrm{R}_{100}\right)$, therapeutic depth $\left(R_{85}\right)$, depth of $50 \%$ isodose $\left(R_{50}\right)$ and the relative surface $\left(D_{\mathrm{s}}\right)$ were taken from the measured and calculated PDD curves. The accuracy of the dose calculation is evaluated by prescribing a known dose of 100 cGy at various SSDs. The plan was exported to the machine and dose was measured using Parallel plate chamber (NACP) using water phantom at the $d_{\max }$. Using TRS 398 protocol [6] the dose was calculated and compared. The isodose distribution calculated by the treatment planning system (TPS) using eMC algorithm perpendicular to the beam central axis were normalized to the half the therapeutic depth $\left(\mathrm{R}_{85}\right)$ taken from the PDD curve. The isodose perpendicular to the beam central axis was measured using I'matriXX device (Scanditronix Wellhofer, Germany) and normalized similar to that of calculated. The planned isodose were transferred to the Omnipro I'matriXX software and compared with the measured isodose. Dose comparison tools such as gamma dose distribution and distance to agreement (DTA) have been used in the analysis [7, 8]. The acceptable gamma pixel parameters were set to $3 \%$ dose and $3 \mathrm{~mm}$ distance-to agreement. The eMC calculated fluence and measured fluence were compared for all the energies and all the cones at 
nominal distance $(\mathrm{SSD}=100 \mathrm{~cm})$ and extended distances $(102,106$, and $110 \mathrm{~cm})$. Based on the fluence comparison the gamma values were estimated by the Omnipro I'matriXX software.

\section{Results}

Figure 1 show the fitted PDD curves for a $10 \times 10 \mathrm{~cm}^{2}$ field size for 6 and $20 \mathrm{MeV}$ electron beam at $100 \mathrm{~cm}$ SSD. The fitted PDD curve is the PDD measured using water phantom and the eMC generated PDD curve. Table 1 shows the comparison between the calculated and measured percentage depth dose data for electron beams of energy $6,9,12,16$ and $20 \mathrm{MeV}$ for $4 \times 4 \mathrm{~cm}^{2}$ cone at nominal and extended SSDs. Table 2 shows the comparison for $10 \times 10 \mathrm{~cm}^{2}$ cone at nominal and extended SSDs.

Table 1: Measured and eMC calculated PDD data comparison for $4 \times 4 \mathrm{~cm}^{2}$ cone.

\begin{tabular}{|c|c|c|c|c|c|c|c|c|c|}
\hline \multirow{2}{*}{ Energy MeV } & \multirow{2}{*}{ SSD cm } & \multicolumn{4}{|c|}{ Measured } & \multicolumn{4}{|c|}{ eMC calculated } \\
\hline & & R100 cm & $\mathbf{R 8 5} \mathrm{cm}$ & $\mathbf{R 5 0} \mathrm{cm}$ & Ds \% & $R 100 \mathrm{~cm}$ & $R 85 \mathrm{~cm}$ & $R 50 \mathrm{~cm}$ & Ds \% \\
\hline \multirow{4}{*}{6} & 100 & 1.4 & 1.98 & 2.49 & 75.8 & 1.3 & 1.93 & 2.45 & 59.7 \\
\hline & 102 & 1.4 & 1.96 & 2.44 & 76.3 & 1.3 & 1.94 & 2.48 & 57.1 \\
\hline & 106 & 1.4 & 1.91 & 2.40 & 77.6 & 1.3 & 1.88 & 2.42 & 65.1 \\
\hline & 110 & 1.4 & 1.95 & 2.45 & 78.1 & 1.2 & 1.80 & 2.35 & 76.1 \\
\hline \multirow{4}{*}{9} & 100 & 2.0 & 2.92 & 3.62 & 82.9 & 2.0 & 2.88 & 3.61 & 74.9 \\
\hline & 102 & 2.0 & 2.87 & 3.58 & 83.4 & 2.0 & 2.86 & 3.61 & 70.4 \\
\hline & 106 & 2.0 & 2.93 & 3.66 & 83.3 & 1.9 & 2.87 & 3.59 & 73.8 \\
\hline & 110 & 2.1 & 2.94 & 3.66 & 83.6 & 2.0 & 2.87 & 3.58 & 70.0 \\
\hline \multirow{4}{*}{12} & 100 & 2.3 & 3.76 & 4.89 & 88.9 & 2.0 & 3.76 & 4.89 & 77.1 \\
\hline & 102 & 2.3 & 3.77 & 4.90 & 88.6 & 2.0 & 3.80 & 4.93 & 74.4 \\
\hline & 106 & 2.3 & 3.86 & 4.95 & 87.8 & 2.0 & 3.85 & 4.98 & 69.4 \\
\hline & 110 & 2.3 & 3.90 & 4.97 & 87.2 & 2.2 & 3.76 & 4.88 & 81.6 \\
\hline \multirow{4}{*}{16} & 100 & 2.1 & 4.47 & 6.10 & 91.9 & 2.2 & 4.49 & 6.18 & 82.5 \\
\hline & 102 & 2.1 & 4.52 & 6.15 & 91.4 & 2.3 & 4.53 & 6.20 & 81.9 \\
\hline & 106 & 2.4 & 4.59 & 6.26 & 90.1 & 2.5 & 4.57 & 6.29 & 80.5 \\
\hline & 110 & 2.3 & 4.62 & 6.32 & 89.4 & 2.8 & 4.62 & 6.25 & 84.9 \\
\hline \multirow{4}{*}{20} & 100 & 1.6 & 5.04 & 7.29 & 93.2 & 1.5 & 5.08 & 7.31 & 91.2 \\
\hline & 102 & 1.9 & 5.13 & 7.35 & 92.2 & 1.8 & 5.32 & 7.45 & 85.4 \\
\hline & 106 & 2.2 & 5.26 & 7.53 & 90.6 & 1.8 & 5.24 & 7.53 & 93.5 \\
\hline & 110 & 2.1 & 5.39 & 7.49 & 90.2 & 1.7 & 5.33 & 7.58 & 89.4 \\
\hline
\end{tabular}

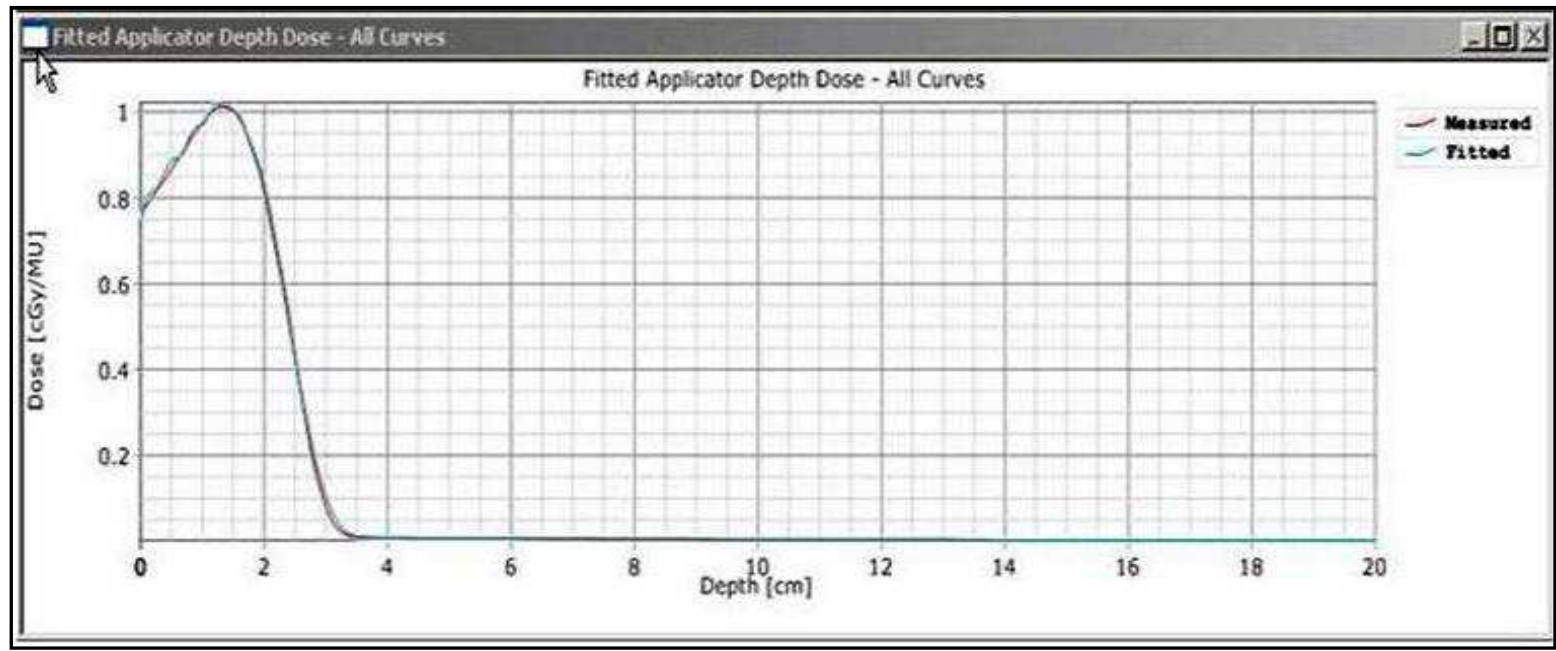

(a) 


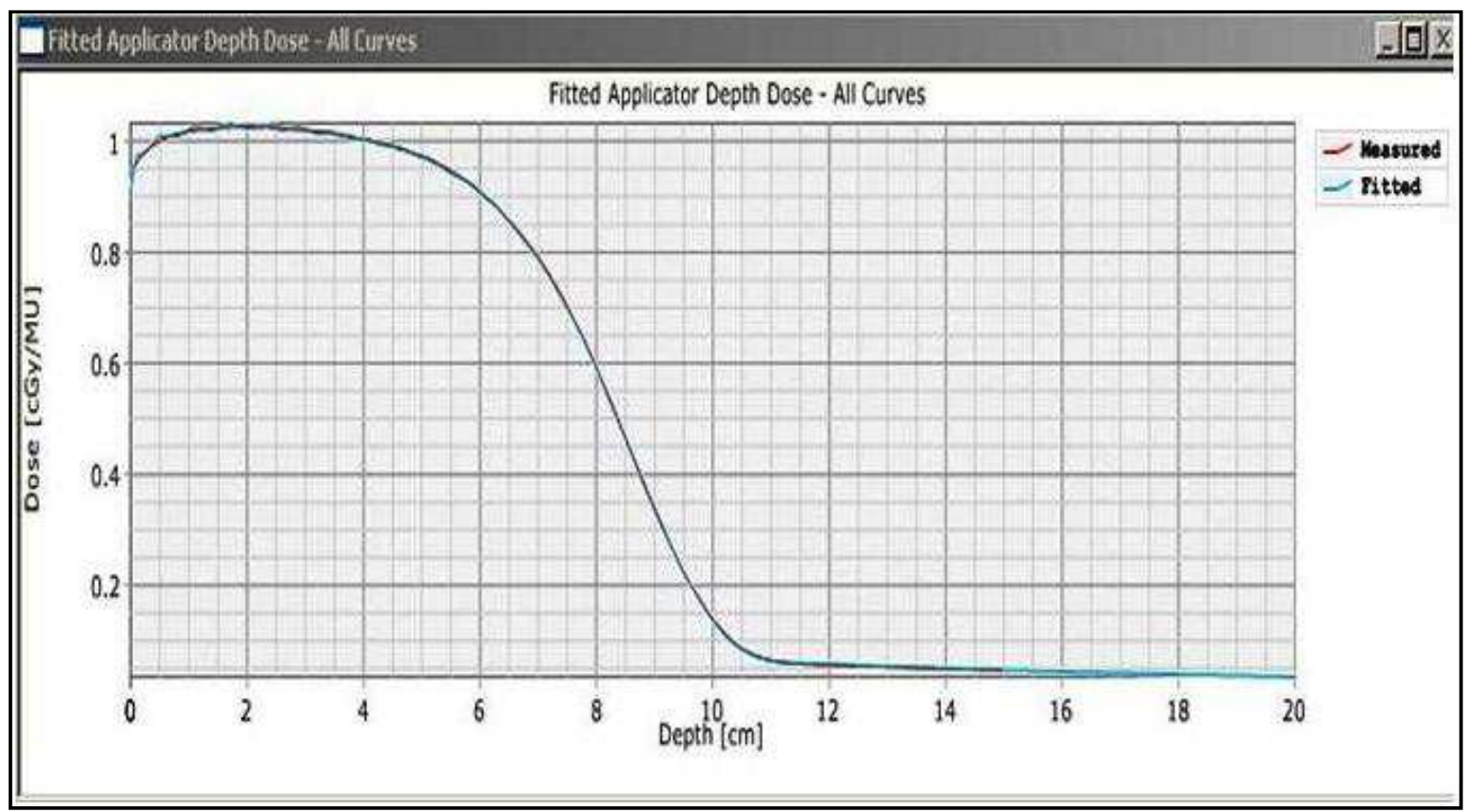

(b)

Figure-1: Measured and eMC calculated PDD curves for $10 \times 10 \mathrm{~cm}^{2}$ at $100 \mathrm{SSD}$ for a) $6 \mathrm{MeV}$ and b) $20 \mathrm{MeV}$ electron beams

Table 2: Measured and eMC calculated PDD data comparison for $10 \times 10 \mathrm{~cm}^{2}$ cone

\begin{tabular}{|c|c|c|c|c|c|c|c|c|c|}
\hline \multirow{2}{*}{ Energy MeV } & \multirow{2}{*}{ SSD cm } & \multicolumn{4}{|c|}{ Measured } & \multicolumn{4}{|c|}{ eMC calculated } \\
\hline & & $R 100 \mathrm{~cm}$ & $R 85 \mathrm{~cm}$ & $R 50 \mathrm{~cm}$ & Ds \% & $R 100 \mathrm{~cm}$ & $R 85 \mathrm{~cm}$ & $R 50 \mathrm{~cm}$ & Ds \% \\
\hline \multirow{4}{*}{6} & 100 & 1.3 & 1.94 & 2.42 & 76.2 & 1.2 & 1.92 & 2.45 & 63.9 \\
\hline & 102 & 1.4 & 2.00 & 2.46 & 75.2 & 1.3 & 1.88 & 2.42 & 68.4 \\
\hline & 106 & 1.4 & 1.95 & 2.41 & 75.0 & 1.2 & 1.92 & 2.45 & 63.8 \\
\hline & 110 & 1.4 & 2.00 & 2.46 & 74.2 & 1.3 & 1.95 & 2.46 & 59.3 \\
\hline \multirow{4}{*}{9} & 100 & 2.2 & 3.01 & 3.70 & 80.5 & 2.2 & 2.95 & 3.68 & 64.3 \\
\hline & 102 & 2.2 & 2.99 & 3.65 & 79.9 & 2.2 & 2.99 & 3.66 & 72.3 \\
\hline & 106 & 2.2 & 3.00 & 3.68 & 79.5 & 2.2 & 3.01 & 3.69 & 71.3 \\
\hline & 110 & 2.2 & 3.05 & 3.72 & 78.4 & 2.2 & 2.99 & 3.68 & 68.3 \\
\hline \multirow{4}{*}{12} & 100 & 2.9 & 4.13 & 5.04 & 86.2 & 3.0 & 4.11 & 5.05 & 71.6 \\
\hline & 102 & 3.0 & 4.19 & 5.08 & 85.2 & 3.0 & 4.18 & 5.08 & 71.7 \\
\hline & 106 & 2.9 & 4.18 & 5.08 & 84.3 & 2.7 & 4.14 & 5.05 & 73.1 \\
\hline & 110 & 3.1 & 4.23 & 5.09 & 83.0 & 2.8 & 4.10 & 5.06 & 76.8 \\
\hline \multirow{4}{*}{16} & 100 & 3.0 & 5.38 & 6.61 & 91.2 & 2.0 & 5.25 & 6.60 & 81.3 \\
\hline & 102 & 3.3 & 5.42 & 6.69 & 90.0 & 2.6 & 5.28 & 6.58 & 88.1 \\
\hline & 106 & 2.7 & 5.46 & 6.67 & 90.7 & 2.6 & 5.32 & 6.58 & 85.9 \\
\hline & 110 & 2.9 & 5.52 & 6.72 & 89.2 & 2.5 & 5.30 & 6.61 & 86.4 \\
\hline \multirow{4}{*}{20} & 100 & 2.0 & 6.35 & 8.29 & 92.8 & 3.2 & 6.31 & 8.26 & 88.6 \\
\hline & 102 & 2.4 & 6.49 & 8.36 & 91.8 & 1.8 & 6.41 & 8.31 & 89.3 \\
\hline & 106 & 2.7 & 6.55 & 8.40 & 89.9 & 2.8 & 6.38 & 8.29 & 80.0 \\
\hline & 110 & 2.9 & 6.60 & 8.42 & 89.9 & 2.0 & 6.49 & 8.35 & 86.7 \\
\hline
\end{tabular}


Table 3: Absolute dose differences between measured and eMC calculated for various field sizes and energies at nominal and extended SSDs

\begin{tabular}{|c|c|c|c|c|c|c|c|}
\hline $\begin{array}{c}\text { Energy } \\
\mathrm{MeV}\end{array}$ & $\begin{array}{c}\text { SSD } \\
\text { Cm }\end{array}$ & $\begin{array}{c}4 \times 4 \\
\mathrm{~cm}^{2}, \%\end{array}$ & $\begin{array}{c}\mathbf{6 x 6} \\
\mathrm{cm}^{2}, \%\end{array}$ & $\begin{array}{c}10 \times 10 \\
\mathrm{~cm}^{2}, \%\end{array}$ & $\begin{array}{c}15 \times 15 \\
\mathrm{~cm}^{2}, \%\end{array}$ & $\begin{array}{c}20 \times 20 \\
\mathrm{~cm}^{2}, \%\end{array}$ & $\begin{array}{l}6.5 \times 9.0 \\
\mathrm{~cm}^{2}, \%\end{array}$ \\
\hline \multirow{4}{*}{6} & 100 & 1.5 & 4.5 & 3.4 & 3.4 & 3.5 & 4.0 \\
\hline & 102 & 0.3 & 2.8 & 2.7 & 3.0 & 3.2 & 2.0 \\
\hline & 106 & 2.3 & 0 & 2.8 & 2.6 & 3.0 & 1.0 \\
\hline & 110 & 4.2 & 1.7 & 2.6 & 2.4 & 3.0 & 1.0 \\
\hline \multirow{4}{*}{9} & 100 & 1.9 & 0.8 & 1.4 & 0.4 & 0.4 & 1.0 \\
\hline & 102 & 2.4 & 0.2 & 1.0 & 0.4 & 2.4 & 1.0 \\
\hline & 106 & 2.9 & 1.7 & 0.8 & 1.1 & 3.0 & 1.0 \\
\hline & 110 & 4.3 & 3.3 & 1.5 & 3.8 & 4.0 & 1.0 \\
\hline \multirow{4}{*}{12} & 100 & 2.9 & 0.4 & 0 & 0 & 1.0 & 3.0 \\
\hline & 102 & 2.1 & 1.6 & 2.8 & 0.8 & 2.0 & 3.0 \\
\hline & 106 & 1.2 & 2.5 & 2.0 & 1.3 & 2.0 & 4.0 \\
\hline & 110 & 1.3 & 3.3 & 2.3 & 1.4 & 3.0 & 4.0 \\
\hline \multirow{4}{*}{16} & 100 & 4.2 & 0.4 & 3.9 & 3.1 & 3.0 & 4.0 \\
\hline & 102 & 4.4 & 1.6 & 2.6 & 3.7 & 4.0 & 3.0 \\
\hline & 106 & 2.2 & 2.5 & 2.4 & 3.6 & 4.0 & 4.0 \\
\hline & 110 & 2.9 & 3.3 & 2.6 & 4.4 & 4.0 & 4.0 \\
\hline \multirow{4}{*}{20} & 100 & 7.6 & 4.2 & 7.0 & 2.5 & 7.0 & 8.0 \\
\hline & 102 & 8.4 & 3.4 & 7.6 & 5.4 & 7.0 & 10. \\
\hline & 106 & 6.2 & 5.0 & 5.9 & 7.2 & 8.0 & 6.0 \\
\hline & 110 & 1.2 & 3.9 & 6.7 & 6.5 & 2.0 & 5.0 \\
\hline
\end{tabular}

Table 4: Gamma value for various field sizes and energies at nominal and extended SSDs

\begin{tabular}{|c|c|c|c|c|c|c|c|}
\hline Energy & SSD & $4 \times 4 \mathrm{~cm}^{2}$ & $6 \times 6 \mathrm{~cm}^{2}$ & $10 \times 10 \mathrm{~cm}^{2}$ & $15 \times 15 \mathrm{~cm}^{2}$ & $20 \times 20 \mathrm{~cm}^{2}$ & $6.5 \times 9.0 \mathrm{~cm}^{2}$ \\
\hline \multirow{4}{*}{6} & 100 & 99.75 & 98.11 & 99.11 & 98.80 & 92.98 & 99.84 \\
\hline & 102 & 99.65 & 100.0 & 98.86 & 98.97 & 99.67 & 99.98 \\
\hline & 106 & 100.0 & 99.85 & 92.64 & 95.16 & 99.21 & 99.94 \\
\hline & 110 & 99.88 & 99.75 & 97.88 & 98.56 & 99.88 & 99.96 \\
\hline \multirow{4}{*}{9} & 100 & 99.67 & 99.23 & 95.12 & 99.98 & 99.93 & 99.86 \\
\hline & 102 & 99.14 & 98.76 & 99.78 & 100 & 99.88 & 99.94 \\
\hline & 106 & 99.49 & 99.70 & 96.76 & 99.97 & 99.96 & 99.94 \\
\hline & 110 & 99.40 & 99.30 & 98.60 & 99.95 & 99.99 & 99.90 \\
\hline \multirow{4}{*}{12} & 100 & 99.93 & 99.80 & 99.17 & 99.61 & 99.95 & 99.95 \\
\hline & 102 & 99.50 & 99.85 & 99.90 & 99.84 & 99.95 & 99.98 \\
\hline & 106 & 99.68 & 98.71 & 97.43 & 99.79 & 99.89 & 99.98 \\
\hline & 110 & 99.68 & 99.89 & 99.57 & 99.97 & 99.95 & 99.98 \\
\hline \multirow{4}{*}{16} & 100 & 99.93 & 99.85 & 95.04 & 99.89 & 99.95 & 99.95 \\
\hline & 102 & 99.31 & 99.97 & 99.98 & 99.88 & 99.89 & 99.99 \\
\hline & 106 & 99.68 & 98.81 & 99.45 & 99.98 & 99.86 & 99.97 \\
\hline & 110 & 99.51 & 99.35 & 99.06 & 99.88 & 99.96 & 99.97 \\
\hline \multirow{4}{*}{20} & 100 & 99.19 & 99.70 & 99.82 & 99.70 & 99.92 & 99.92 \\
\hline & 102 & 98.90 & 99.30 & 99.97 & 99.92 & 99.82 & 99.77 \\
\hline & 106 & 99.49 & 99.97 & 99.42 & 99.99 & 99.99 & 99.95 \\
\hline & 110 & 99.30 & 99.87 & 99.88 & 99.78 & 99.83 & 99.99 \\
\hline
\end{tabular}




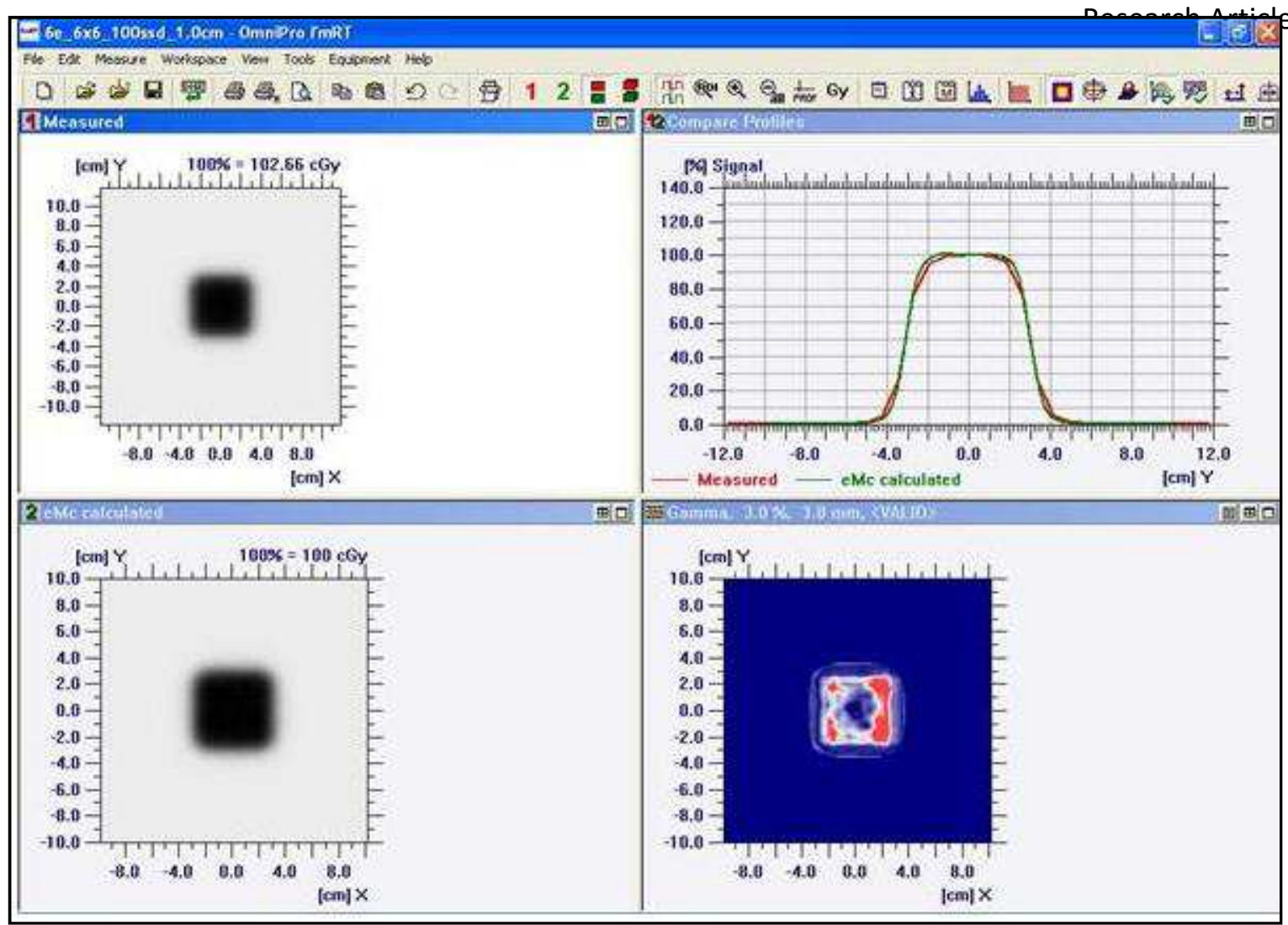

(a)

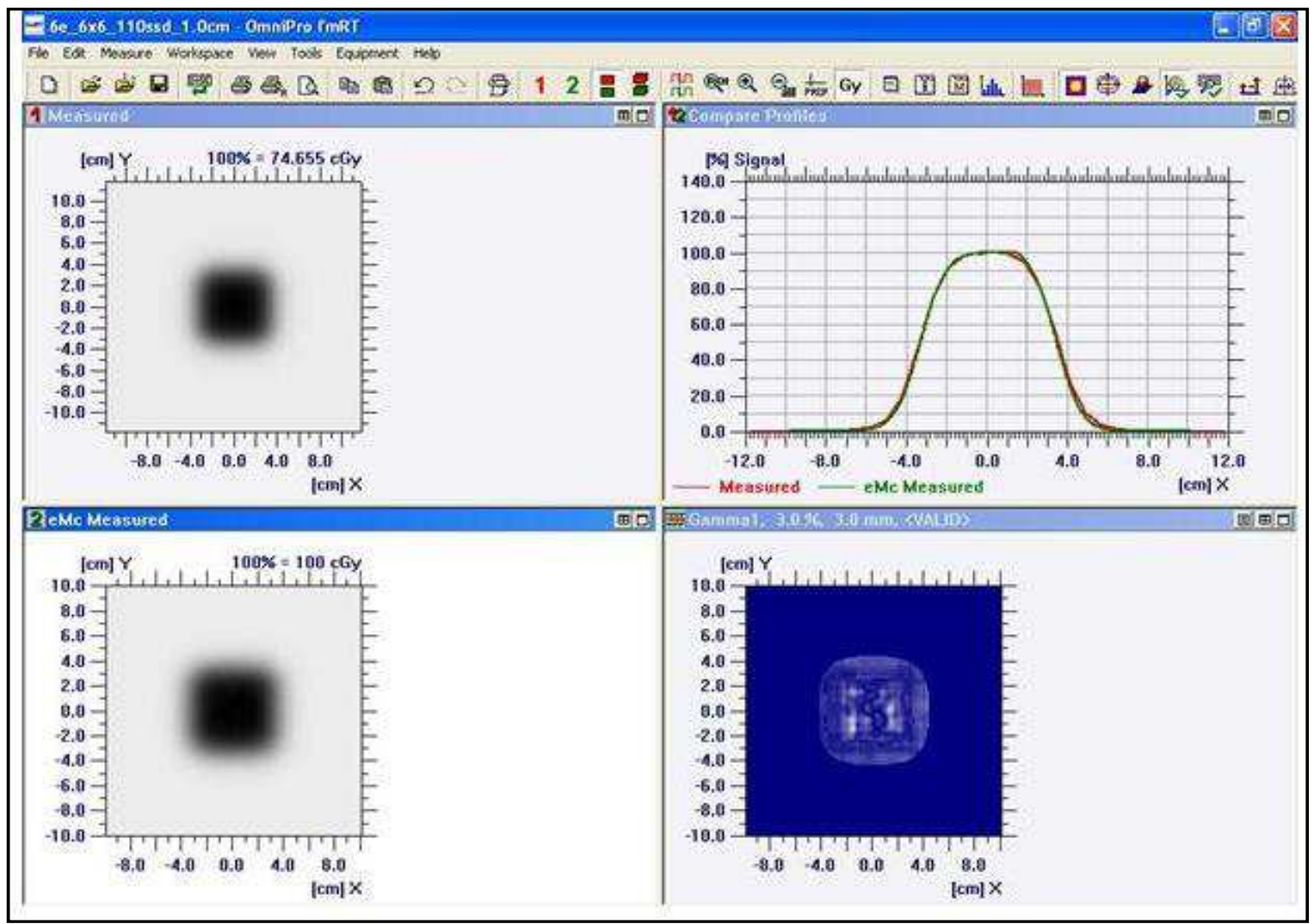

(b)

Figure-2: Comparison of measured and calculated fluence for $10 \times 10 \mathrm{~cm}^{2}$ regular field size at a) $100 \mathrm{SSD}$ b) $110 \mathrm{SSD}$ 


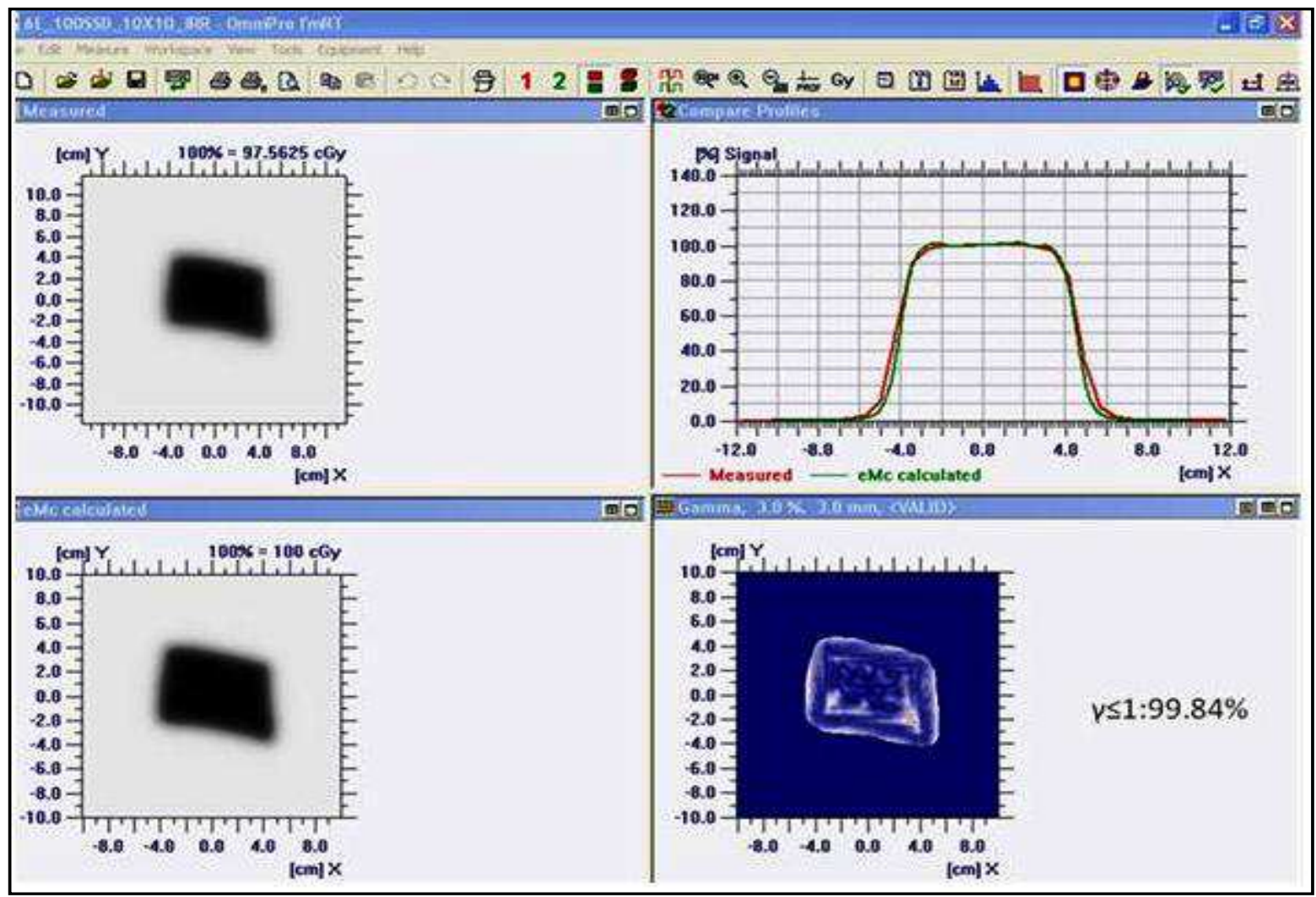

(a)

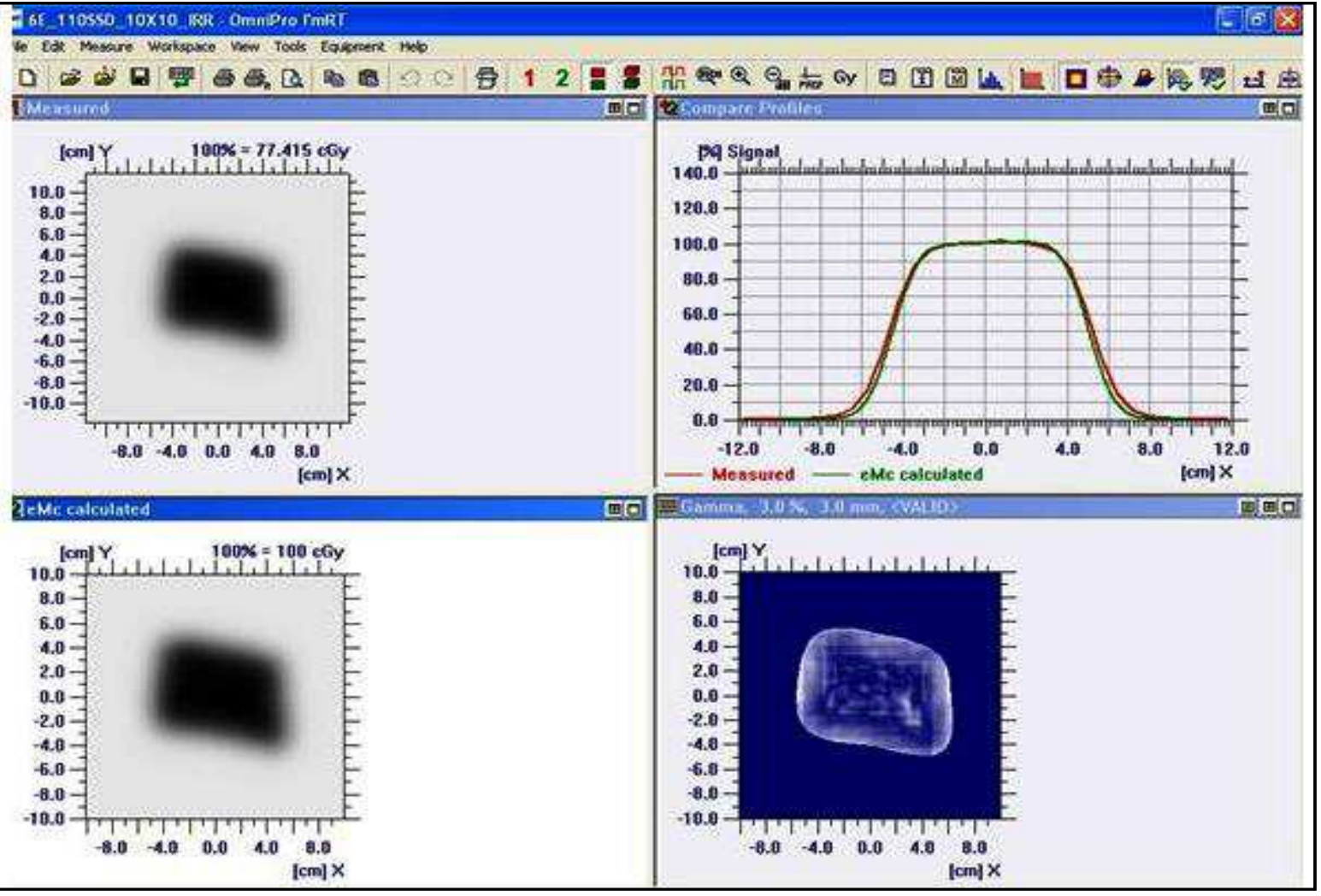

(b)

Figure-3: Comparison of measured and calculated fluence for $6.5 \times 9 \mathrm{~cm}^{2}$ irregular field size at a) $100 \mathrm{SSD}$ b) $110 \mathrm{SSD}$ 
The eMC plans display surface dose by interpolating the dose at the first grid point inside the phantom and the first grid point outside the phantom, resulting in the surface under dose. Since for higher energies the maximum dose peak was broad there was a maximum of 10 and $12 \mathrm{~mm}$ shift observed with 16 and $20 \mathrm{MeV}$ for $10 \times 10 \mathrm{~cm}^{2}$ field size. Table 3 shows the comparison of prescribed dose and the measured dose for all the electron energies and for all the cone sizes at nominal and extended SSDs. The agreement of dose was within 5\% for 6, 9, 12 and $16 \mathrm{MeV}$ for all field sizes. But for 20 $\mathrm{MeV}$ it has gone up to $8 \%$. This was in agreement with the findings reported by Ding et al [4]. Figure 2 shows the gamma analysis for measured and eMC calculated fluence for a regular field $\left(10 \times 10 \mathrm{~cm}^{2}\right)$ at 100 and $110 \mathrm{~cm}$ SSD. Figure 3 shows the gamma analysis for the measured and eMC calculated isodose for an irregular field $\left(6.5 \times 9 \mathrm{~cm}^{2}\right)$ at 100 and $110 \mathrm{~cm}$ SSD. Table 4 shows the gamma values for $4 \times 4 \mathrm{~cm}^{2}, 6 \times 6 \mathrm{~cm}^{2}, 10 \times 10 \mathrm{~cm}^{2}, 15 \times 15 \mathrm{~cm}^{2}, 20 \times 20 \mathrm{~cm}^{2}$ and for irregular field size $6.5 \times 9 \mathrm{~cm}^{2}$ (used for patient treatment).

\section{Discussion}

Based on our results in Table 1 and 2 , For $4 \times 4 \mathrm{~cm}^{2}$ field size the maximum shift in $\mathrm{R}_{100}$ was $5 \mathrm{~mm}, \mathrm{R}_{85}$ was $1.9 \mathrm{~mm}, \mathrm{R}_{50}$ was $0.9 \mathrm{~mm}$ and the variation in the relative surface $\left(D_{S}\right)$ was about $25 \%$. For $10 \times 10 \mathrm{~cm}^{2}$ field size the maximum shift in $\mathrm{R}_{100}$ was $12 \mathrm{~mm}, \mathrm{R}_{85}$ was $2.2 \mathrm{~mm}, \mathrm{R}_{50}$ was $1.1 \mathrm{~mm}$ and the variation in the relative surface $\left(D_{S}\right)$ was about $20 \%$. The plot of PDD from eMC plans overlaid with those of the measurements show good agreements except for the first 1 to $2 \mathrm{~mm}$ of the surface.

In a similar study by $\mathrm{Xu}$ et al [3] they have compared eMC calculations and measurements of depth doses, isodose distributions, and monitor units for several different energy and small field cutout size combinations at different SSDs. Their results indicate that the eMC algorithm can accurately predict depth doses, isodose distributions, and monitor units (within $2.5 \%$ ) for field sizes as small as $3.0 \mathrm{~cm}$ diameter for energies in the 6 to $20 \mathrm{MeV}$ range at $100 \mathrm{~cm} \mathrm{SSD}$. Their results were in consistent with the recommendation of Popple et al [9].

Yang et al. [10] have commissioned electron Monte Carlo (eMC) algorithm in Eclipse Treatment Planning System (TPS) for TrueBeam Linacs, including the evaluation of dose calculation accuracy for small fields and oblique beams and comparison with the existing eMC model for Clinacs. Pemler et al. [11] have evaluated the commercial electron beam treatment planning system on the basis of a Monte Carlo algorithm (Varian Eclipse, eMC V7.2.35). They have Measured dose distributions were used for comparison with dose distributions predicted by eMC calculations and the tests were carried out for various applicators and field sizes, irregular shaped cut outs and an inhomogeneity phantom for energies between $6 \mathrm{Me} \mathrm{V}$ and $22 \mathrm{MeV}$ Monitor units were calculated for all applicator/energy combinations and field sizes down to
$3 \mathrm{~cm}$ diameter and source-to-surface distances of 100 $\mathrm{cm}$ and $110 \mathrm{~cm}$.

Ya et al [12] Commissioned the eMC algorithm on multiple identical linacs provided a unique opportunity to systematically evaluate the algorithm with actual measurements of clinically relevant beam and dose parameters. They have measured and eMC calculated dose distributions were compared both along and perpendicular to electron beam direction for electron energy/applicator/depth combination using measurement data from four Varian 21EX CLINAC linear accelerator and their results indicate that eMC algorithm in Eclipse provides acceptable agreement with measurement data for most clinical situations. The gamma analysis results show excellent agreements with greater than $98 \%$ of the pixels passing the gamma requirements.

Chamberland et al. [13] studied the accuracy of the electron Monte Carlo (eMC) dose calculation algorithm included in a commercial treatment planning system and compare its performance against an electron pencil beam algorithm. From their results eMC algorithm showed good agreements with the measurements in simple homogeneous and heterogeneous phantoms. Compared to the electron pencil beam algorithms, the eMC calculations predicted more accurately large dose perturbations due to inhomogeneities. The eMC algorithm can be considered for routine treatment planning. In our present study results in the Table 4 and Figures 2 and 3 show excellent agreements with greater than $98 \%$ of the pixels passing the gamma requirements.

\section{Conclusion}

In conclusion the comparison of eMC calculations and measurements for various field sizes and energies shows that the Monte Carlo algorithm for electron 
planning is more accurate. The eMC algorithm performs well in a homogeneous water phantom with regular and irregular fields at nominal and extended SSD.

Author's disclosure: We have no disclosures to be made.

Funding: Nil, Conflict of interest: None initiated. Permission from IRB: Yes

\section{References}

1. Neunschwander H, Mackie TR, Reckwerdt PJ. MMC-a high-performance Monte carlo code for electron beam treatment planning. Phys Med Biol.1995;40(4):543-74.

2. Popple RA, Weinber R, Antolok JA, et al. Comprehensive evaluation of a commercial macro Monte carlo electron dose calculation implementation using a standard verification data set. Med Phys. 2006;33(6):1540-51.

3. Xu Z, Walsh SE, Telivala TP, Meek AG Yang G. Evaluation of the eclipse electron Monte Carlo dose calculation for small fields. J. Appl. Clin. Med. Phy 2009; 10(3): 75-85.

4. Ding GX, Duggan DM, Coffey CW, Shokrani P, Cygler JE. First Monte Carlo based commercial dose calculation module for the electron beam treatment planning-new issues for clinical consideration. Phys Med Biol.2006;51(11):2781-99.

5. Eclipse beam data collection guide. Experts from Eclipse Integrated treatment planning "Reference guide for algorithms" March 2004.
6. International code of practice for Dosimetry based on absorbed dose to water. IAEA Tech. series No 398, Absorbed dose determination in external beam radiotherapy. IAEA, Vienna (2000).

7. Low DA, Dempsey JF. Evaluation of the gamma dose distribution comparison method. Med Phys. 2003;30(9):2455-64.

8. Low DA, Harms WB, Mutic S, Purdy JA. A technique for the quantitative evaluation of the dose distributions. Med Phys. 1998;25(5):656-61.

9. Popple RA, Weinber R, Antolak JA, et al. Comprehensive evaluation of a commercial macro Monte Carlo electron dose calculation implementation using a standard verification data set. Med Phys. 2006;33(6):1540-51.

10. Yang $\mathrm{X}, \mathrm{G}$ Lasio $^{1}, \mathrm{~J} \mathrm{Zhou}^{1}, \mathrm{M} \mathrm{Lin}{ }^{1}, \mathrm{~B} \mathrm{Yi}^{1}$ and $\mathrm{M}$ Guerrero ${ }^{1}$ Commissioning of Electron Monte Carlo in Eclipse Treatment Planning System for TrueBeam . Med. Phys. 2014; 41, 362-6.

11. Pemler P, Besserer J, Schneider U, Neuenschwander H. Evaluation of a commercial electron treatment planning system based on Monte Carlo techniques (eMC). Z Med Phys. 2006;16(4):313-29.

12. Hu A, Song H, Chen Z, Zhou S, Yin FF. Evaluation of an electron Monte Carlo dose calculation algorithm for electron beam. J Appl Clin Med Phys.2009;10(2),115.

13. Chamberland E, Beaulieu L, Lachance B. Evaluation of an electron Monte Carlo dose calculation algorithm for treatment planning. J Appl Clin Med Phys. 2015;16(3), 60-79.

\section{How to cite this article?}

T. Arunkumar, C. Varatharaj, M. Ravikumar, K. M. Ganesh, S. Sathiyan, Shwetha B. Commissioning and validation of the electron Monte Carlo dose calculation at extended source to surface distance from a medical linear accelerator. Int $J$ Med Res Rev 2016;4 (7):1155-1163.doi: 10.17511/ijmrr.2016.i07.15. 